\title{
Broadband and band-limited random vibration energy harvesting using a piezoelectric patch on a thin plate
}

\author{
Ugur Aridogan*a ${ }^{*}$ Ipek Basdogan ${ }^{\mathrm{a}}$, Alper Erturk ${ }^{\mathrm{b}}$ \\ ${ }^{a}$ Department of Mechanical Engineering, College of Engineering, \\ Koç University, Istanbul, Turkey, 34450; \\ ${ }^{\mathrm{b}} \mathrm{G}$. W. Woodruff School of Mechanical Engineering, \\ Georgia Institute of Technology, Atlanta, GA, USA, 30332-0405
}

\begin{abstract}
This paper presents analytical modeling and case studies of broadband and band-limited random vibration energy harvesting using a piezoceramic patch attached on a thin plate. The literature of vibration-based energy harvesting has been mostly focused on resonant cantilevered structures. However, cantilevered beam-type harvesters have limited broadband vibration energy harvesting capabilities unless they are combined with a nonlinear component. Moreover, cantilever arrangements cannot always be mounted on thin structures (which are basic components of marine, aerospace, and ground transportation systems) without significantly affecting the host system's design and overall dynamics. A patch-based piezoelectric energy harvester structurally integrated to a thin plate can be a proper alternative to using resonant cantilevers for harvesting energy from thin structures. Besides, plate-like structures have more number of vibration modes compared to beam structures, offering better broadband performance characteristics. In this paper, we present analytical modelling of patch-based piezoelectric energy harvester attached on a thin plate for random vibrations. The analytical model is based on electromechanically-coupled distributed-parameter formulation and validated by comparing the electromechanical frequency response functions (FRFs) with experimental results. Example case studies are then presented to investigate the expected power output of a piezoceramic patch attached on an aluminum plate for the case of random force excitations. The effect of bandwidth of random excitation on the mean power and shunted mean-square vibration response are explored with a focus on the number of vibration modes covered in the frequency range of input power spectral density (PSD).
\end{abstract}

Keywords: Piezoelectricity, Energy Harvesting, Random Vibration, Electromechanical Modelling

\section{INTRODUCTION}

Vibrational energy harvesting has been extensively studied over the past decade for taking advantage of ambient mechanical energy ${ }^{1-3}$. The driving force of the vibrational energy harvesting research is to power small electronic devices such as wireless sensor networks and eliminate the need of battery replacement and disposal. The conversion of vibration-to-electric energy has been demonstrated with electrostatic ${ }^{4}$, electromagnetic 5 , piezoelectric ${ }^{6}$, magnetostrictive materials ${ }^{7}$, and electroactive polymers ${ }^{8}$. Among these conversion alternatives, piezoelectric energy harvesting has been most heavily research due to their high power density and ease of application ${ }^{3}$.

Research efforts in the field of piezoelectric energy harvesting have been mostly focused on cantilevered beam-type harvesters. Electromechanically-coupled analytical ${ }^{9}$, semi-analytical ${ }^{10}$, and numerical models ${ }^{11}$ of resonant harvesters have been developed and experimentally validated under harmonic base excitations for a wide range of electrical loads. Despite the extensive research efforts on harmonic base excitation of resonant harvesters, rather limited research has considered non-harmonic or stochastic broadband random excitations. A number of authors have studied broadband random vibrations of cantilevered beam harvesters with lumped-parameter ${ }^{12-15}$ and distributed-parameter ${ }^{16}$ modeling. Random excitation of monostable and bistable nonlinear energy harvesters have also been explored ${ }^{17,18}$. A patch-based piezoelectric energy harvester attached on a thin plate can be a reasonable alternative to resonant beam harvesters in order not to modify the host plate dynamics and design substantially. Plate-like structures have more number of modes over a low-frequency range, yielding better broadband energy harvesting capabilities. Recently, analytical model of an

*uaridogan@ku.edu.tr 
energy harvester in the form of a piezoelectric patch attached to a thin plate was presented for energy harvesting from thin plates by Aridogan et al. ${ }^{19}$ and the current work implements that model to broadband and band-limited random excitation cases.

This paper presents the distributed-parameter modeling and case studies of broadband and band-limited random vibration energy harvesting using a piezoceramic patch attached on a thin plate. The effect of bandwidth of random excitation on the mean power and shunted mean-square vibration response is explored with a focus on the number of vibration modes covered in the frequency range of the input power spectral density. In the following, distributedparameter modelling of a patch-based harvester attached on a thin plate is formulated for random vibration energy harvesting. Analytical predictions of electromechanical frequency response functions are experimentally validated for a wide range of resistive load. Finally analytical case studies of broadband and band-limited vibrations are given to investigate the performance of patch-based harvesters.

\section{DISTRIBUTED-PARAMETER ELECTROELASTIC MODELLING}

\subsection{Governing Equations in Physical Coordinates}

Figure 1 presents a patch-based piezoelectric energy harvester attached on a thin plate excited by a transverse point force $f(t)$. A transversely isotropic piezoceramic patch having a length of $l_{p}$ and a width of $w_{p}$ is perfectly bonded on the plate whose length and width are $l_{s}$ and $w_{s}$. The thicknesses of the plate $\left(h_{s}\right)$ and harvester patch $\left(h_{p}\right)$ are assumed to be very small compared to the other dimensions to justify the thin plate assumption of the Kirchhoff plate theory. It is assumed that negligibly thin electrode layers cover surfaces of the harvester patch and a load resistance $R_{l}$ is considered as an electrical load connected to the bottom and top electrodes.

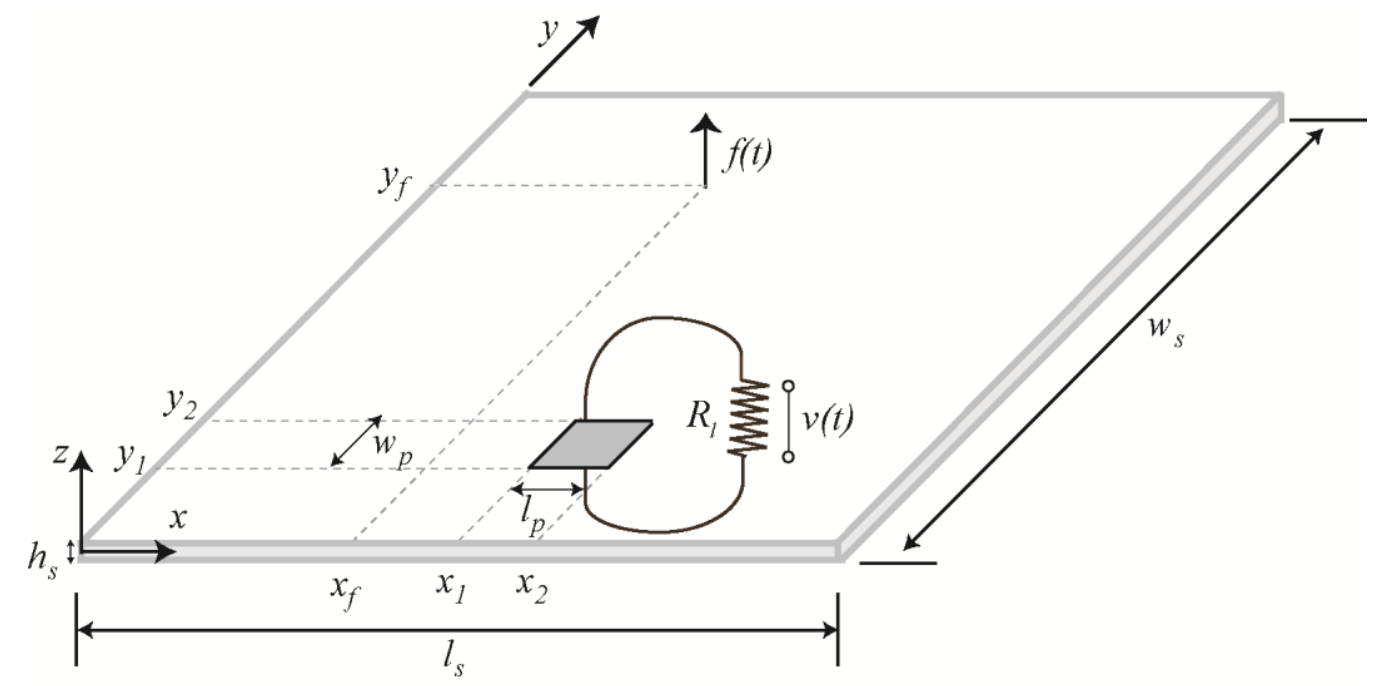

Figure 1. Piezoelectric patch-based energy harvester attached on a thin plate under point-force excitation

The electromechanically-coupled equation of motion of the plate together with a harvester patch is ${ }^{19}$

$$
D\left(\frac{\partial^{4} w(x, y, t)}{\partial x^{4}}+2 \frac{\partial^{4} w(x, y, t)}{\partial x^{2} \partial y^{2}}+\frac{\partial^{4} w(x, y, t)}{\partial y^{4}}\right)+c_{a} \frac{\partial w(x, y, t)}{\partial t}+\rho_{s} h_{s} \frac{\partial^{2} w(x, y, t)}{\partial t^{2}}-\kappa_{p} \theta_{p} v(t)=f(t) \delta\left(x-x_{0}\right) \delta\left(y-y_{0}\right)
$$

where the deflection of the neutral surface of the plate is $w(x, y, t)$ at position $(x, y)$ and time $t$. The voltage across the resistive load is $v(t)$. The flexural rigidity, Poisson's ratio, density of the plate are $D, v_{s}$ and $\rho_{s}$, respectively. The flexural rigidity can be obtained by $D=Y_{s} h_{s}^{3} /\left(12-12 v_{s}^{2}\right)$ where $Y_{s}$ is the Young's modulus of the plate. The viscous air damping coefficient is $c_{a}$. The electromechanical coupling term for the harvester patch is $\theta_{p}=h_{p c} \bar{e}_{31}$ where the reference surface is $h_{p c}$ and the effective piezoelectric constant is $\bar{e}_{31}$. The induced moment coefficient $\kappa_{p}$ is 


$$
\kappa_{p}=\left[\frac{\mathrm{d} \delta\left(x-x_{1}\right)}{\mathrm{d} x}-\frac{\mathrm{d} \delta\left(x-x_{2}\right)}{\mathrm{dx}}\right]\left[H\left(y-y_{1}\right)-H\left(y-y_{2}\right)\right]+\left[\frac{\mathrm{d} \delta\left(y-y_{1}\right)}{\mathrm{dy}}-\frac{\mathrm{d} \delta\left(y-y_{2}\right)}{\mathrm{dy}}\right]\left[H\left(x-x_{1}\right)-H\left(x-x_{2}\right)\right]
$$

Here, $\delta(x)$ and $\delta(y)$ are the Dirac delta functions, $H(x)$ and $H(y)$ are the Heaviside functions along $x$ and $y$ axes, respectively. It should be noted that the piezoelectrically induced moments are taken into account as a result of the converse piezoelectric effect, while the inertial and end effects of the harvester patch are neglected.

The electrical circuit equation governing electrical dynamics of the harvester patch shown in figure 1 is given by ${ }^{19}$

$$
C_{p} \frac{\mathrm{d} v(t)}{\mathrm{d} t}+\frac{v(t)}{R_{l}}+\theta_{p}\left\{\int_{y=y_{1}}^{y_{2}} \int_{x=x_{1}}^{x_{2}}\left[\frac{\partial^{3} w(x, y, t)}{\partial x^{2} \partial t}+\frac{\partial^{3} w(x, y, t)}{\partial y^{2} \partial t}\right] \mathrm{d} x \mathrm{~d} y\right\}=0
$$

where the capacitance of the harvester patch is $C_{p}=\left(\bar{\varepsilon}_{33} w_{p} l_{p}\right) / h_{p}$ and $\bar{\varepsilon}_{33}$ is the permittivity component at constant strain $S$. Equations (1) and (3) constitute the electromechanically-coupled distributed parameter model of the piezoceramic patch-based harvester attached on a thin plate. Simultaneous solution of these two equations will yield the voltage output across the resistive load and the vibration response of the plate.

\subsection{Modal Transformation and Electromechanical FRFs}

The transverse vibration of a thin plate can be expressed in terms of mass normalized eigenfunctions $\phi_{m n}(x, y)$ and modal mechanical response $\eta_{m n}(t)$ as

$$
w(x, y, t)=\sum_{n=1}^{\infty} \sum_{m=1}^{\infty} \phi_{m n}(x, y) \eta_{m n}(t)
$$

for the $m n$-th vibration mode. By following standard modal analysis procedure, second-order ordinary differential equation for the modal mechanical response $\eta_{m n}(t)$ can be derived as

$$
\frac{\mathrm{d}^{2} \eta_{m n}(t)}{\mathrm{d} t^{2}}+2 \zeta_{m n} \omega_{m n} \frac{\mathrm{d} \eta_{m n}(t)}{\mathrm{d} t}+\omega_{m n}^{2} \eta_{m n}(t)-\tilde{\theta}_{m n} v(t)=f_{m n}(t)
$$

Here, $\omega_{m n}$ is the undamped natural frequency and $\zeta_{m n}$ is the damping ratio for the $m n$-th vibration mode. The electromechanical coupling term and point-force excitation are $\widetilde{\theta}_{m n}$ and $f_{m n}$ in modal coordinates ${ }^{19}$. The electrical circuit equation in modal coordinates can be obtained as follows:

$$
C_{p} \frac{\mathrm{d} v(t)}{\mathrm{d} t}+\frac{v(t)}{R_{l}}+\sum_{n=1}^{\infty} \sum_{m=1}^{\infty} \widetilde{\theta}_{m n} \frac{\mathrm{d} \eta_{m n}(t)}{\mathrm{d} t}=0
$$

Based on the linear system assumptions, if the transverse point-force acting on the plate is harmonic of the form $f(t)=F_{0} e^{\mathrm{j} \omega t}$ (where $F_{0}$ and $\omega$ are the amplitude and frequency of the force and $j$ is the unit imaginary number), the steady-state modal mechanical and voltage responses can be expressed as $\eta_{m n}(t)=\mathrm{H}_{m n} e^{\mathrm{j} \omega t}$ and $v(t)=V e^{\mathrm{j} \omega t}$ where $\mathrm{H}_{m n}$ and $V$ are complex amplitudes. By substituting these steady-state expressions into equation (5) and (6), two electromechanical FRFs can be extracted between two outputs (i.e., voltage and vibration response) and one input (i.e., point-force excitation). The voltage FRF $\alpha(\omega)$ relates the voltage output to point force input as

$$
\alpha(\omega)=\frac{v(t)}{F_{0} e^{j \omega t}}=\frac{V}{F_{0}}
$$


and the displacement FRF relates the vibration response to point force input as

$$
\beta(x, y, \omega)=\frac{w(x, y, t)}{F_{0} e^{j \omega t}}=\frac{1}{F_{0}} \sum_{n=1}^{\infty} \sum_{m=1}^{\infty} \mathrm{H}_{m n} \phi_{m n}(x, y) .
$$

\subsection{Mean Power Output and Mean-Square Vibration Response}

Having formulated the electromechanical FRFs, the expected value of the power output (mean power) can be expressed as

$$
E[P(t)]=\int_{-\infty}^{\infty} \frac{S_{0}}{R_{l}}|\alpha(\omega)|^{2} d \omega
$$

if the point-force excitation is assumed to be ideal Gaussian white noise having a constant power spectral density (PSD) value $S_{0}$ in the entire frequency band. Similarly, the mean-square value of the vibration response is

$$
E\left[w^{2}(x, y, t)\right]=\int_{-\infty}^{\infty} S_{0}|\beta(\omega, x, y)|^{2} d \omega
$$

in the displacement form. For the case of band-limited strongly ergodic random excitation over the frequency range $[-\bar{\omega}, \bar{\omega}]$, the expected value of the power output can be written as

$$
E[P(t)]=\int_{-\bar{\omega}}^{\bar{\omega}} \frac{S_{f}(\omega)}{R_{l}}|\alpha(\omega)|^{2} d \omega
$$

and the mean-square vibration is

$$
E\left[w^{2}(x, y, t)\right]=\int_{-\bar{\omega}}^{\bar{\omega}} S_{f}(\omega)|\beta(\omega, x, y)|^{2} d \omega
$$

where $S_{f}(\omega)$ is the frequency-dependent PSD. It should be noted that the upper frequency limit $\bar{\omega}$ must be set to a high value such that the vibration modes contributing to the power output and vibration response are covered.

\section{EXPERIMENTAL VALIDATION}

\subsection{Experimental Setup}

In the experimental setup presented in figure 2, an isotropic piezoceramic patch (T105-A4E-602 by Piezo Systems, Inc.) is employed as a patch-based piezoelectric energy harvester by perfectly bonding on an all-edges-clamped aluminum thin plate. Geometric, material and dielectric properties of the aluminum plate and piezoceramic patch are provided in Table 1. The bottom and top surfaces of the harvester patch are covered by thin vacuum sputtered nickel electrodes and an electrical load (simply a resistor) is connected to the bottom and top electrode terminals. An electromechanical shaker is used to excite the plate with a sine-sweep signal generated by a signal generator and amplified by a high voltage amplifier (see the shaker's location in figure 2). For force measurements, a force transducer (PCB 208C01) is placed between the armature of the shaker and the plate surface. A laser vibrometer (Polytec PDV 100) is used to measure the transverse vibration of the plate at the center of the harvester patch. The signals from the force transducer, laser vibrometer and voltage across the resistor are recorded and analyzed with a data acquisition system. Experimental FRFs between the voltage output-to-force input (voltage FRF) and vibration response-to-force input (velocity FRF) are obtained with the signal analyzer software. 


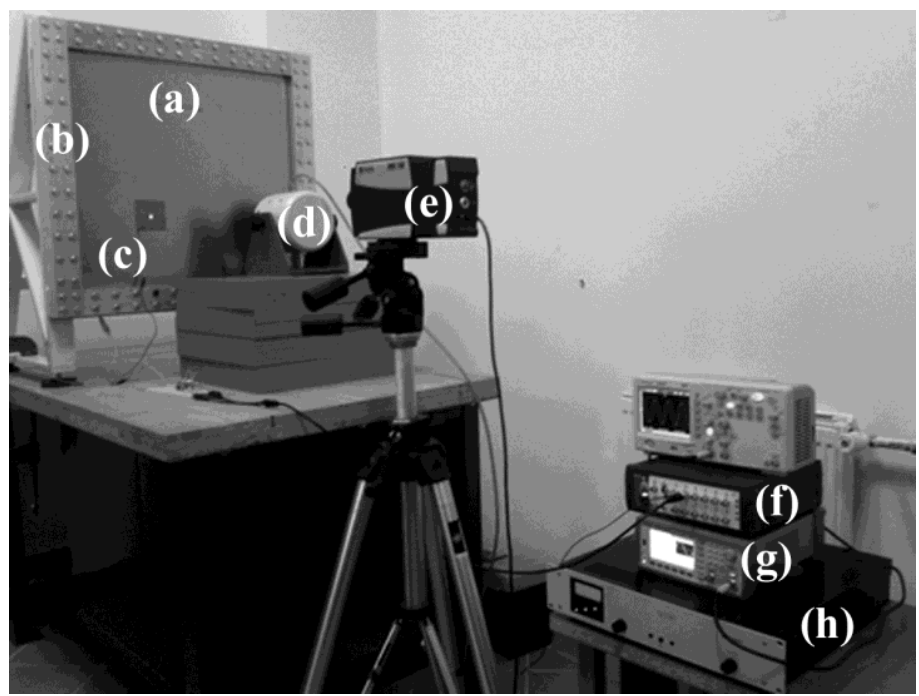

Figure 2. Experimental setup: (a) Aluminum plate (b) clamping frame (c) patch-based harvester (d) shaker (e) laser vibrometer (f) data acquisition unit ( $\mathrm{g}$ ) signal generator $(\mathrm{h})$ high voltage amplifier

Table 1. Geometric, material and dielectric properties

\begin{tabular}{|c|c|c|}
\hline Property & Aluminum & Piezoceramic \\
\hline Length $(\mathrm{mm})$ & 580 & 72.4 \\
\hline Width $(\mathrm{mm})$ & 540 & 72.4 \\
\hline Thickness $(\mathrm{mm})$ & 1.96 & 0.267 \\
\hline Young's modulus $(\mathrm{GPa})$ & 70 & 66 \\
\hline Mass density $\left(\mathrm{kg} \mathrm{m}^{-3}\right)$ & 2700 & 7800 \\
\hline Piezoelectric constant $d_{31}\left(\mathrm{pm} \mathrm{V}^{-1}\right)$ & - & -190 \\
\hline Permittivity constant $\bar{\varepsilon}_{33}^{S}\left(\mathrm{nF} \mathrm{m}^{-1}\right)$ & - & 10.38 \\
\hline
\end{tabular}

\subsection{Electromechanical Frequency Response Functions (FRFs)}

Electromechanical FRFs are experimentally measured using the experimental setup (presented in figure 2) and estimated using the electromechanically-coupled distributed parameter model based on the properties given in Table 1. Figure 3 presents the analytical prediction and experimental measurement of velocity FRF for a resistive load of $1 \mathrm{M} \Omega$ (close to open-circuit conditions). Note that, the modal damping ratios used in the analytical model are extracted from the experimental measurement shown in figure 3 by applying the half-power point method at each resonance frequency. It is clear from this figure that the analytical velocity FRF accurately predicts the experimental measurement near resonance and anti-resonance frequencies. The experimental and analytical voltage FRFs are presented in figure 4 for a set of three resistors with values of $470 \Omega, 1.18 \mathrm{k} \Omega, 1 \mathrm{M} \Omega$. As can be seen in figure 4, analytical and experimental voltage FRFs are in very good agreement for a wide range of resistive loads. Hence, it can be concluded that experimental electromechanical FRFs can be accurately predicted using analytical model in the bandwidth from $25 \mathrm{~Hz}$ to $250 \mathrm{~Hz}$.

In the next section, analytical case studies are given based on electromechanical FRF formulation validated here to investigate the mean power output and mean-square vibration response for the case of band-limited random force excitations up to $250 \mathrm{~Hz}$. 


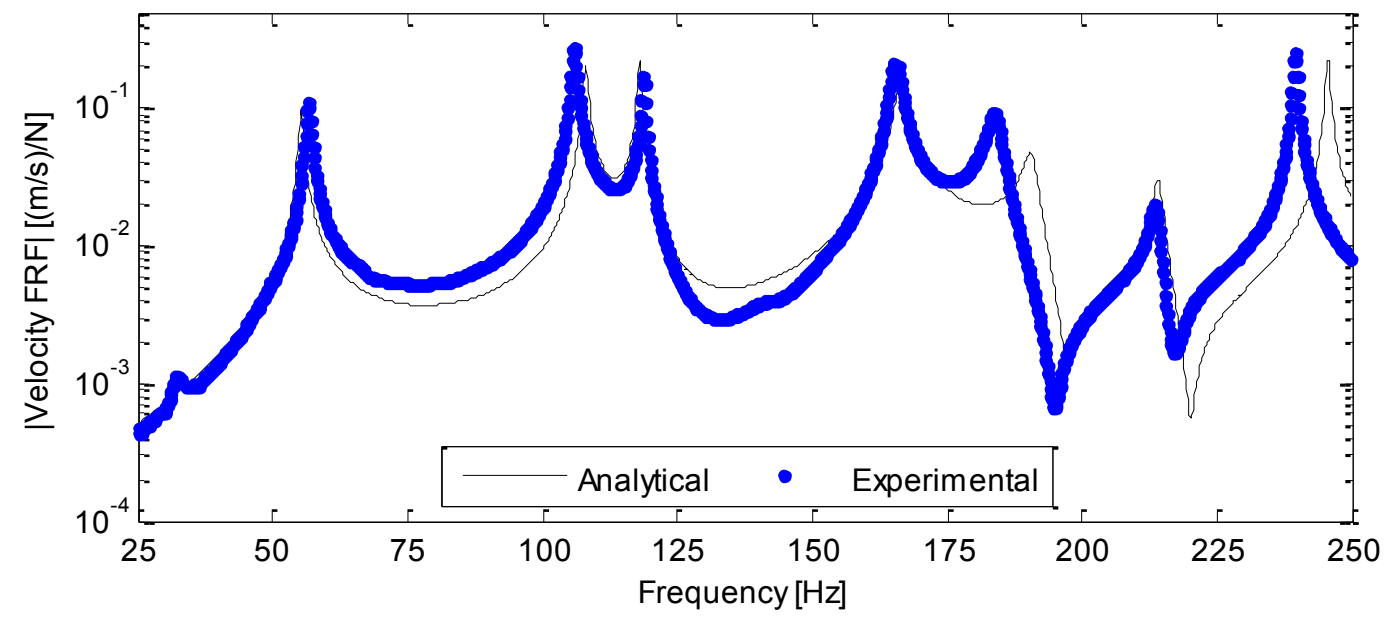

Figure 3. Comparison of the experimental and analytical velocity FRFs for a resistor load of $1 \mathrm{M} \Omega$

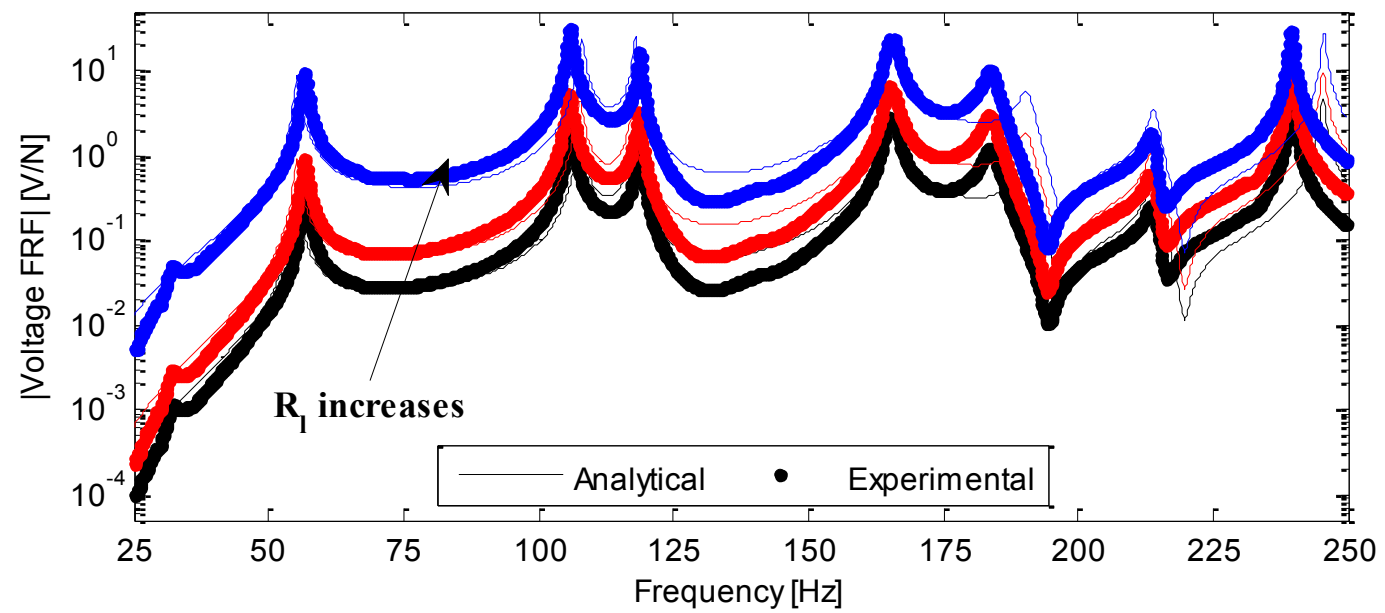

Figure 4. Comparison of the experimental and analytical voltage FRFs for a set of resistor loads (470 $\Omega, 1.18 \mathrm{k} \Omega, 1 \mathrm{M} \Omega$ )

\section{ANALYTICAL CASE STUDIES}

\subsection{Case Studies for Band-limited Random Vibrations}

To study the effect of bandwidth of random excitation on the mean power output and shunted mean-square vibration response, four different random force excitation signals are simulated in which the frequency band covers particular vibration modes of the thin plate. A sample time history of each of these excitation signals and their corresponding PSD profiles are presented in figure 5. It is important to note that standard deviations of four signals are identical and equal to $50 \mathrm{mN}(0.05 \mathrm{~kg} \mathrm{~m} / \mathrm{s} 2)$ while their mean values are zero. As can be observed from PSD profiles in figure 5, the first excitation signal covers the frequency range between $25 \mathrm{~Hz}$ to $75 \mathrm{~Hz}$. The second and third signals have the flat PSD levels in the frequency ranges from $75 \mathrm{~Hz}$ to $150 \mathrm{~Hz}$ and from $150 \mathrm{~Hz}$ to $250 \mathrm{~Hz}$, respectively. The PSD of the fourth signal is uniformly distributed over the whole $25-250 \mathrm{~Hz}$ band. Note that the average level of flat portions of PSD profiles is also given in figure 5 . 
(a)
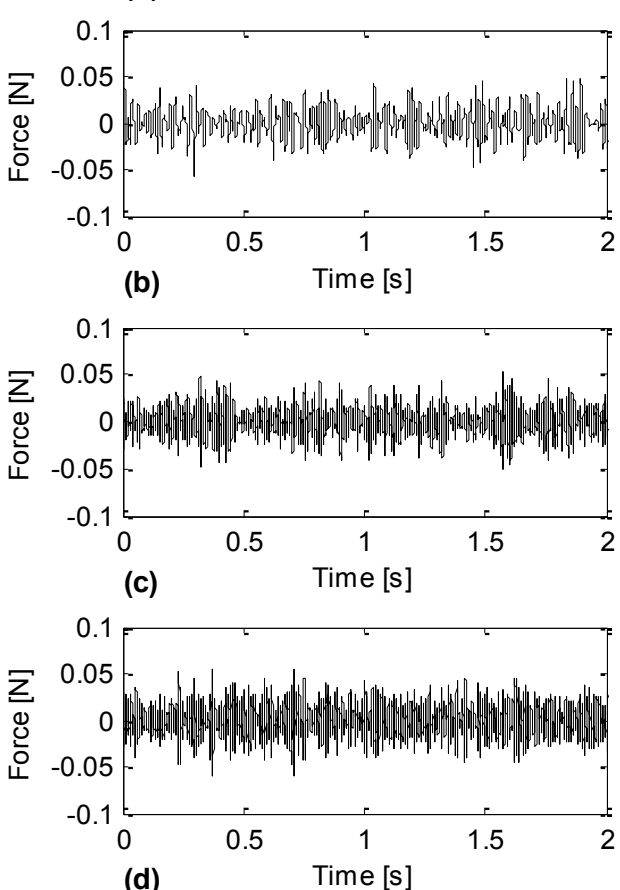

(d)

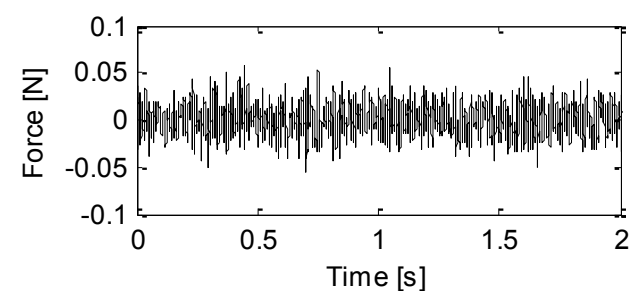

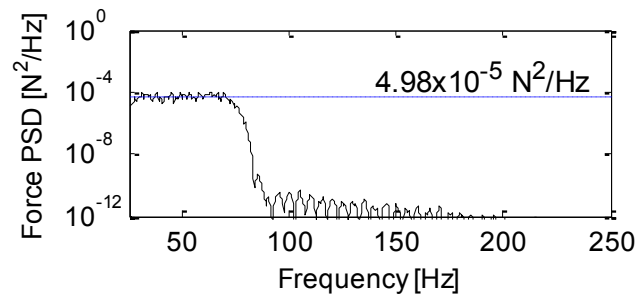
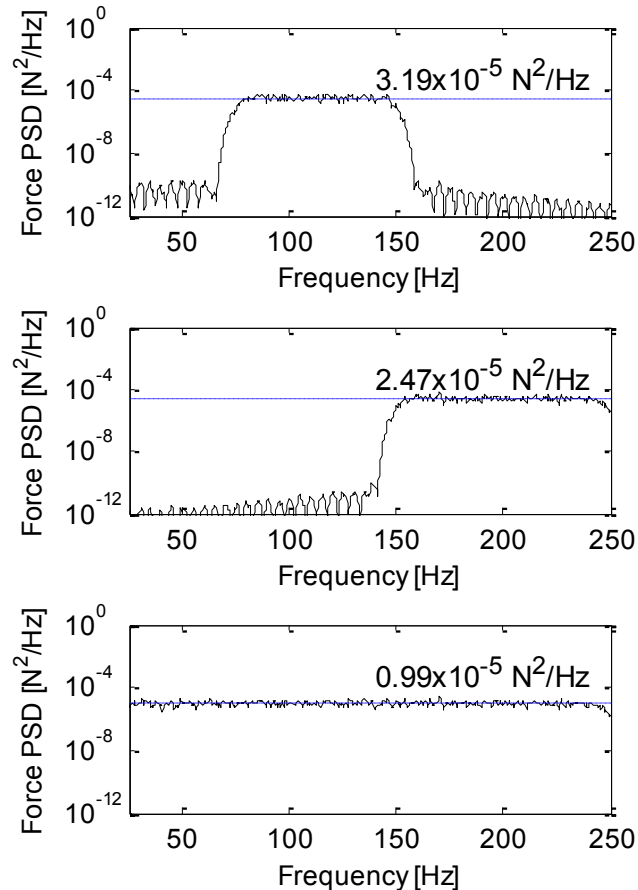

Figure 5. A sample time history and corresponding PSD profiles of the four different force excitation signals in the bandwidth of (a) $25-75 \mathrm{~Hz}$ (b) $75-150 \mathrm{~Hz}$ (c) $150-250 \mathrm{~Hz}$ (d) $25-250 \mathrm{~Hz}$ (standard deviations of the time signals are identical)

For the four cases of the force excitation signal, the expected power output and mean-square velocity responses are analytically obtained and presented in figure 6 by performing a resistor sweep from $100 \Omega$ to $1 \mathrm{M} \Omega$. Comparing the expected power output of four different band-limited force excitations having identical standard deviations, it can be noticed that the force excitation in the bandwidth from $150 \mathrm{~Hz}$ to $250 \mathrm{~Hz}$ produces significantly more maximum expected power output at the optimum resistive load. The reason for extracting more power in this band is due to the excitation of the five vibration modes of the thin plate in the corresponding frequency range and vibration-mode dependent performance of the harvester patch ${ }^{19}$. Noting that the fourth excitation signal's PSD is uniformly distributed over the total band from $25 \mathrm{~Hz}$ to $250 \mathrm{~Hz}$, its maximum mean power output is less than the one for the force excitation in the bandwidth of $150 \mathrm{~Hz}$ to $250 \mathrm{~Hz}$. One should note that the input PSD levels are not identical (figure 5(b)) in this comparison that uses the same standard deviation for the time signals (figure 5(a)). As can be observed from figure 6(b), the mean-square vibration response has the higher values for band-limited random excitation in the frequency range from $75 \mathrm{~Hz}$ to $150 \mathrm{~Hz}$ as compared to other three excitation signals. It can also be noticed that the optimum resistive load for the maximum mean power output roughly corresponds to the maximum vibration attenuation for all four cases. Another comparison can be made by taking the band-limited PSD values (rather than the standard deviations of time series) to be the same as discussed next. 
(a)

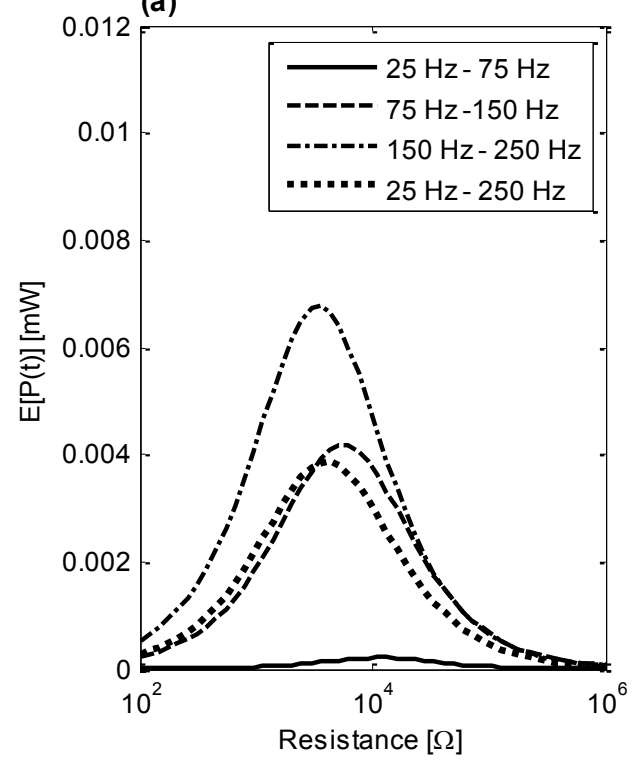

(b)

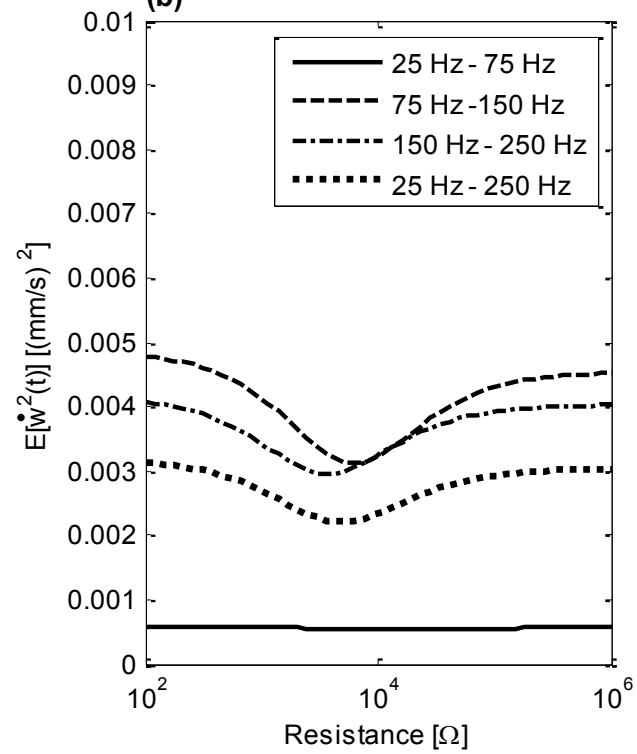

Figure 6. Comparison of the four different band-limited force excitation signals in terms of (a) mean power output vs. resistive load (b) mean-square velocity response vs. resistive load (standard deviations of the time signals are identical)

For a PSD-based comparison of the four different band-limited force excitation signals, the expected mean power output and shunted mean-square velocity response are normalized with respect to the flat PSD levels provided in figure 5. The normalized power outputs and vibration responses are plotted in figure 7. It can be observed from this figure that the normalized mean power output and mean-square velocity response have the highest value for the excitation signal in the bandwidth of $25 \mathrm{~Hz}$ to $250 \mathrm{~Hz}$, which excites all the vibration modes of the host structure up to $250 \mathrm{~Hz}$.

(a)

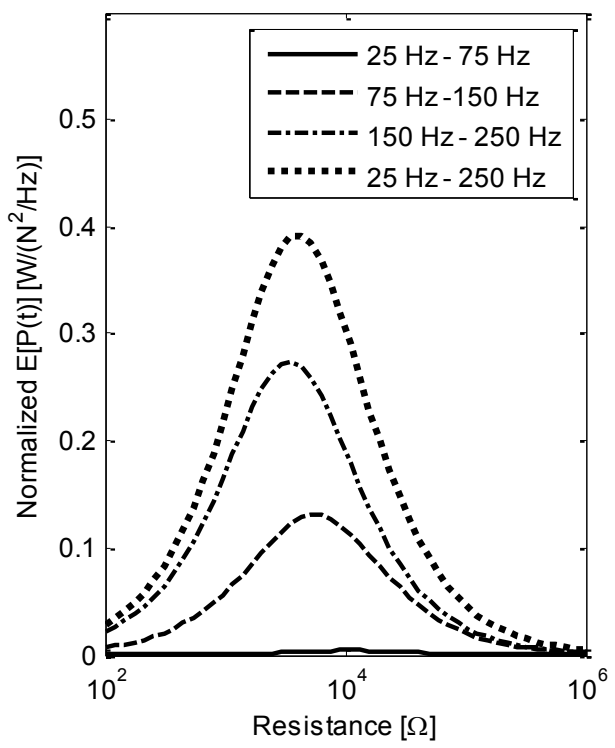

(b)

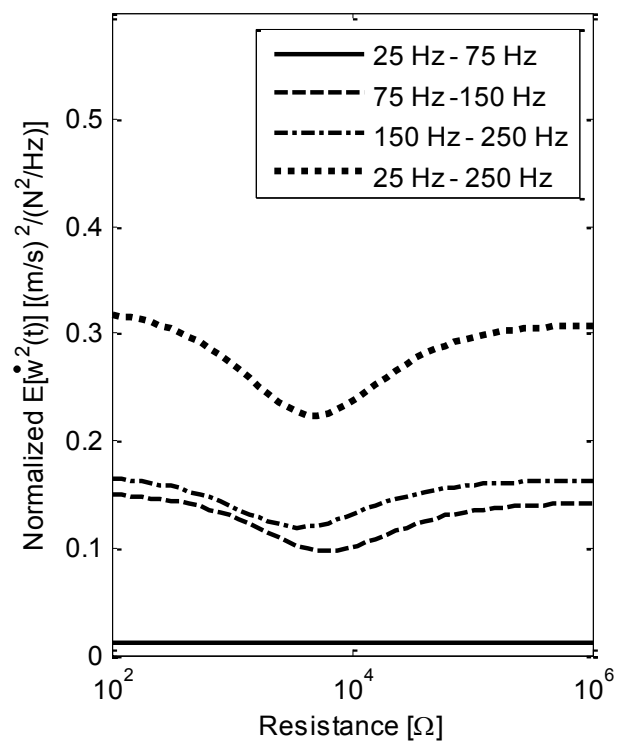

Figure 7. Comparison of the four different band-limited force excitation signals in terms of: (a) normalized mean power output vs. resistive load (b) normalized mean-square velocity response vs. resistive load (results are normalized with respect to PSD levels) 


\subsection{Optimal Positioning of the Patch-based Piezoelectric Energy Harvester}

In this section, the variation of the expected power output with the position of the harvester patch is analyzed in case of the band-limited force excitation. Analytical random vibrational energy harvesting simulations for the different positions of the harvester patch are carried out to predict the expected power output for a wide range of resistive load and then the maximum mean power output is extracted by determining the optimum resistive load. Note that, the center of the harvester patch (with the dimensions of 72.4 X $72.4 \mathrm{~mm}$ ) is varied over an area $40 \mathrm{~mm}$ in from the clamped ends.

Before analyzing the simulation results, it is worth to explore the strain mode shapes of the plate between 0 and $250 \mathrm{~Hz}$. As pointed out by Aridogan et al. ${ }^{19}$, a patch-based energy harvester can generate much larger power output at the vibration modes where there is a homogenous strain field under the patch. Therefore, for the host plate shown in figure 2 , the analytical first eight undamped natural frequencies and normalized strain fields are presented in figure 8 .

Based on the simulation results, the maximum mean power output of the harvester patch at different locations on the plate is determined and presented in figure 9. As can be seen from this figure, optimal location of the harvester patch depends on the bandwidth of the force excitation signal. For example, the bandwidth from $25 \mathrm{~Hz}$ to $75 \mathrm{~Hz}$ excites only the first vibration mode of the host plate and the optimal location is found to be the center of the host plate where the corresponding strain mode shape is the maximum as can be also noticed from figure 8 . The second excitation signal (in the bandwidth from $75 \mathrm{~Hz}$ to $150 \mathrm{~Hz}$ ) excites the second and third vibration modes, and the optimal location is found to be near the quadrants of the host plate (it can be also depicted from figure 8). For the case of excitation signals (in the bandwidth from $150 \mathrm{~Hz}$ to $250 \mathrm{~Hz}$ and from $25 \mathrm{~Hz}$ to $250 \mathrm{~Hz}$ ) that excite the higher vibration modes of the host plate, the optimal location of the harvester patch moves the intersection of intermediate strain fields, which can be observed by examining both of figures 8 and 9 .

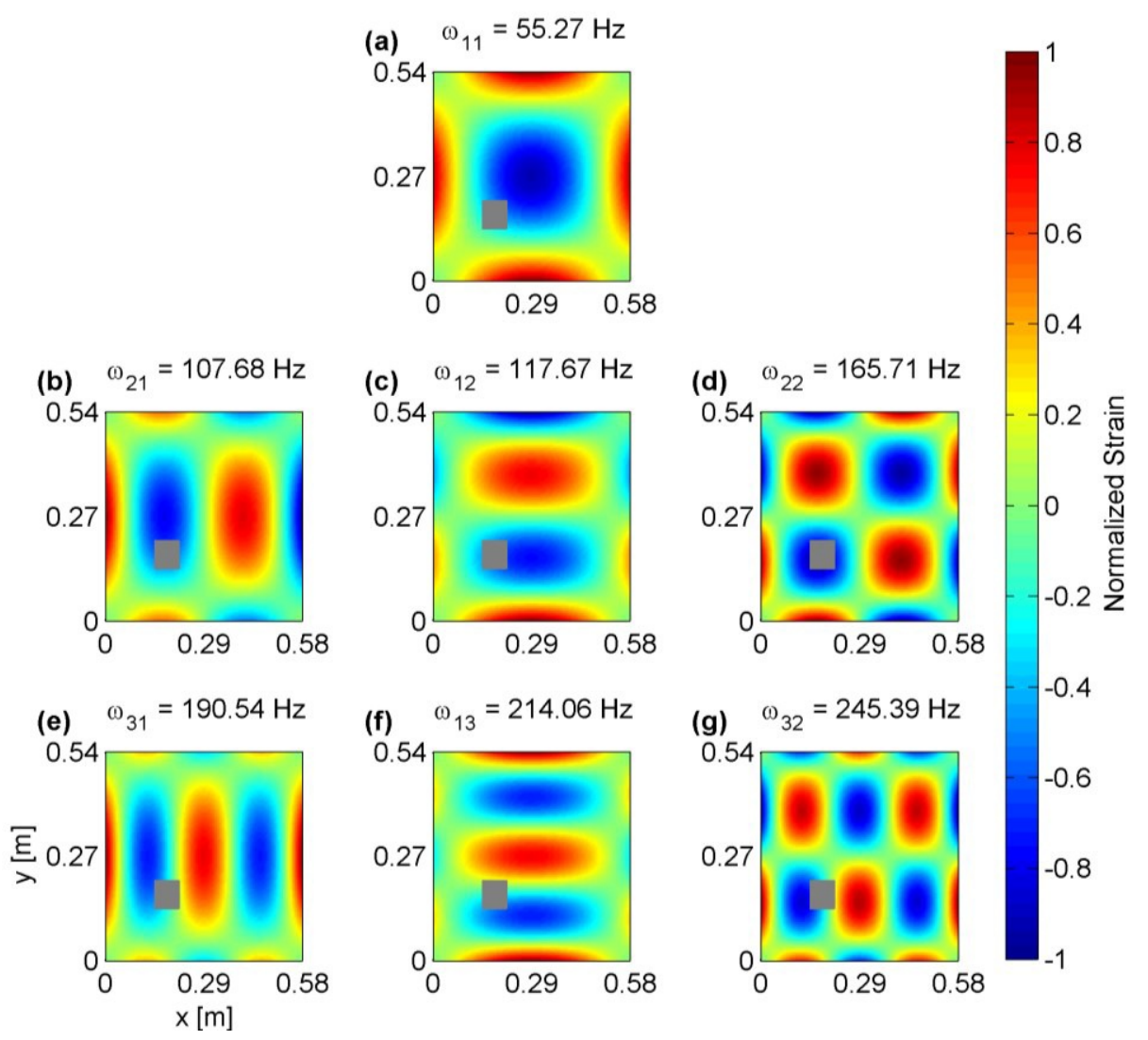

Figure 8. Normalized strain fields for the (a) first, (b) second, (c) third, and (d) fourth (e) fifth (f) sixth (g) seventh mode of the CCCC plate with the gray shaded area covered by the harvester patch in the experimental validation. 


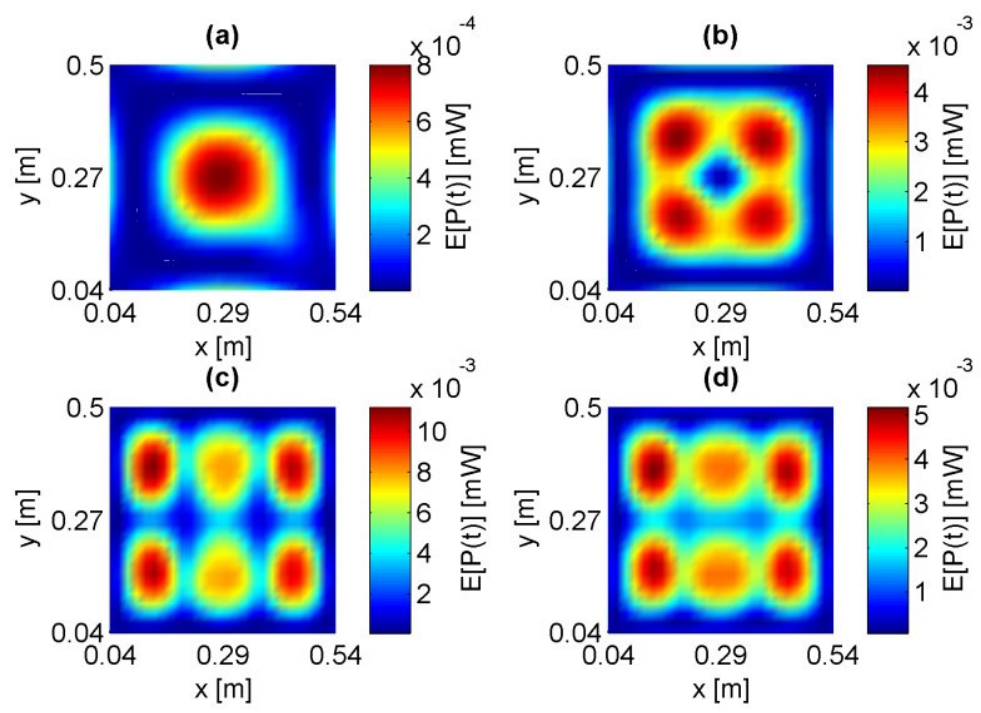

Figure 9. Expected power output at different patch-based harvester positions for the force excitation in the frequency range: (a) $25-75 \mathrm{~Hz}$ (b) $75-150 \mathrm{~Hz}$ (c) $150-250 \mathrm{~Hz}$ (d) $25-250 \mathrm{~Hz}$

\section{CONCLUSION}

A patch-based piezoelectric energy harvester is a reasonable alternative to cantilevered beam harvesters for harvesting energy from structural vibrations of thin plates without modifying the plate dynamics and design substantially. The distributed-parameter modeling and case studies of broadband and band-limited random vibration energy harvesting using a piezoceramic patch attached on a thin plate were studied in this paper. The electromechanical FRF predictions of the analytical model were validated by experimental results. The effect of bandwidth of random excitation on the mean power and shunted mean-square vibration response was analytically investigated with four different band-limited force excitations. The variation of the expected power output with the position of the harvester patch was also analyzed for all band-limited force excitation cases. It is shown that the optimal location of the harvester patch depends on the bandwidth of the force excitation signal and the modeling framework presented here can be utilized for optimal positioning of the harvesters on plate-like structures for broadband and band-limited random vibration energy harvesting.

\section{ACKNOWLEDGMENTS}

Support from the Koç University TÜPRAŞ Energy Center (KÜTEM) is gratefully acknowledged.

\section{REFERENCES}

[1] S. P. Beeby, M. J. Tudor, and N. M. White, "Energy harvesting vibration sources for microsystems applications," Measurement Science \& Technology, 17(12), R175-R195 (2006).

[2] S. R. Anton, and H. A. Sodano, "A review of power harvesting using piezoelectric materials (2003-2006)," Smart Materials \& Structures, 16(3), R1-R21 (2007).

[3] K. A. Cook-Chennault, N. Thambi, and A. M. Sastry, "Powering MEMS portable devices-a review of nonregenerative and regenerative power supply systems with special emphasis on piezoelectric energy harvesting systems," Smart Materials and Structures, 17(4), 043001 (2008).

[4] C. Lee, Y. M. Lim, B. Yang et al., "Theoretical comparison of the energy harvesting capability among various electrostatic mechanisms from structure aspect," Sensors and Actuators A: Physical, 156(1), 208-216 (2009).

[5] S. P. Beeby, R. N. Torah, M. J. Tudor et al., "A micro electromagnetic generator for vibration energy harvesting,” Journal of Micromechanics and Microengineering, 17(7), 1257 (2007).

[6] A. Erturk, and D. J. Inman, [Piezoelectric Energy Harvesting] John Wiley \& Sons, Ltd, Chichester(2011). 
[7] L. Wang, and F. G. Yuan, "Vibration energy harvesting by magnetostrictive material," Smart Materials and Structures, 17(4), 045009 (2008).

[8] J. Brufau-Penella, M. Puig-Vidal, P. Giannone et al., "Characterization of the harvesting capabilities of an ionic polymer metal composite device," Smart Materials and Structures, 17(1), 015009 (2008).

[9] A. Erturk, and D. J. Inman, "A Distributed Parameter Electromechanical Model for Cantilevered Piezoelectric Energy Harvesters," Journal of Vibration and Acoustics, 130(4), 041002-15 (2008).

[10] A. Erturk, "Assumed-modes modeling of piezoelectric energy harvesters: Euler-Bernoulli, Rayleigh, and Timoshenko models with axial deformations," Computers \& Structures, 106-107(0), 214-227 (2012).

[11] G. Wang, "Analysis of bimorph piezoelectric beam energy harvesters using Timoshenko and Euler-Bernoulli beam theory," Journal of Intelligent Material Systems and Structures, 24(2), 226-239 (2013).

[12] M. Ferrari, V. Ferrari, M. Guizzetti et al., "Improved Energy Harvesting from Wideband Vibrations by Nonlinear Piezoelectric Converters," Procedia Chemistry, 1(1), 1203-1206 (2009).

[13] J. T. Scruggs, "An optimal stochastic control theory for distributed energy harvesting networks," Journal of Sound and Vibration, 320(4-5), 707-725 (2009).

[14] D. A. W. Barton, S. G. Burrow, and L. R. Clare, "Energy Harvesting From Vibrations With a Nonlinear Oscillator," Journal of Vibration and Acoustics, 132(2), 021009-021009 (2010).

[15] L. C. J. Blystad, E. Halvorsen, and S. Husa, "Piezoelectric MEMS energy harvesting systems driven by harmonic and random vibrations," Ultrasonics, Ferroelectrics and Frequency Control, IEEE Transactions on, 57(4), 908-919 (2010).

[16] S. Zhao, and A. Erturk, "Electroelastic modeling and experimental validations of piezoelectric energy harvesting from broadband random vibrations of cantilevered bimorphs," Smart Materials and Structures, 22(1), $015002(2013)$.

[17] M. F. Daqaq, "Response of uni-modal duffing-type harvesters to random forced excitations," Journal of Sound and Vibration, 329(18), 3621-3631 (2010).

[18] S. Zhao, and A. Erturk, "On the stochastic excitation of monostable and bistable electroelastic power generators: Relative advantages and tradeoffs in a physical system," Applied Physics Letters, 102(10), 103902 (2013).

[19] U. Aridogan, I. Basdogan, and A. Erturk, "Analytical modeling and experimental validation of a structurallyintegrated piezoelectric energy harvester on a thin plate," Smart Materials and Structures, In Press, (2014). 